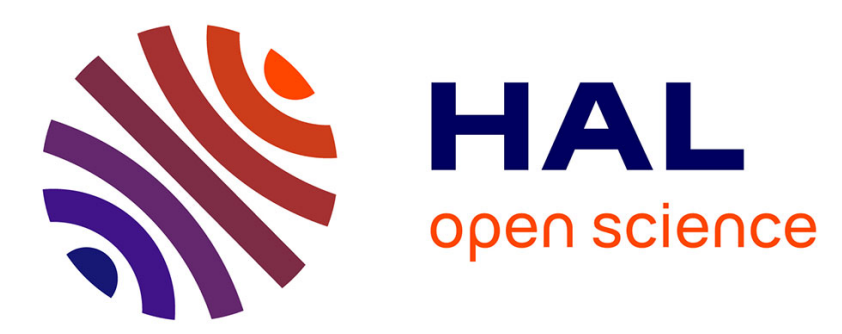

\title{
Direct Numerical Simulation of flow instabilities over Savonius style wind turbine blades
} Antoine Ducoin, Mostafa Safdari Shadloo, Sukanta Roy

\section{To cite this version:}

Antoine Ducoin, Mostafa Safdari Shadloo, Sukanta Roy. Direct Numerical Simulation of flow instabilities over Savonius style wind turbine blades. Renewable Energy, 2017, 105, pp.374-385. 10.1016/j.renene.2016.12.072 . hal-01611150

\author{
HAL Id: hal-01611150 \\ https://hal.science/hal-01611150
}

Submitted on 17 Jun 2019

HAL is a multi-disciplinary open access archive for the deposit and dissemination of scientific research documents, whether they are published or not. The documents may come from teaching and research institutions in France or abroad, or from public or private research centers.
L'archive ouverte pluridisciplinaire HAL, est destinée au dépôt et à la diffusion de documents scientifiques de niveau recherche, publiés ou non, émanant des établissements d'enseignement et de recherche français ou étrangers, des laboratoires publics ou privés. 
1. Direct Numerical Simulation of Flow Instabilities over Savonius Style Wind Turbine Blades

\author{
A. Ducoin ${ }^{1}$, M. S. Shadloo ${ }^{2}$ and S. Roy ${ }^{3}$ \\ ${ }^{1}$ LHEEA laboratory, Ecole Centrale de Nantes (CNRS UMR 6598), 44300 Nantes, \\ France \\ ${ }^{2}$ CORIA Laboratory-UMR 6614, CNRS-University 83 INSA of Rouen, 76000, Rouen, \\ France \\ ${ }^{3}$ IRPHE Laboratory UMR 7342, Aix Marseille University, France
}

9 Abstract

In this paper, Direct Numerical Simulations (DNS) are carried out in order to capture the flow instabilities and transition to turbulence occurring on a Savonius style wind turbine (SSWT) blade. Simulations are conducted with the open source code NEK5000, solving the incompressible Navier-Stokes equations with a high order, spectral element method. Because of the relatively high Reynolds number considered $\left(R e_{\xi}=9 \times 10^{4}\right)$, the computational domain of the Savonius blade is reduced to the pressure side, and the blade is studied in static condition, which avoids the large scale vortex shedding that occurs on its suction side, particularly allows to investigate the static performance of the wind turbine. The results suggest that Görtler vortices can occur and cause the flow to transit to turbulence, which modify the pressure and wall friction distributions, and consequently alter the drag and lift forces.

Keywords: Direct Numerical Simulation, Savonius Style Wind Turbine, centrifugal instability, blade performance

\footnotetext{
${ }^{1}$ antoine.ducoin@ec-nantes.fr
} 


\section{Introduction}

The objective of this paper is to investigate the flow instability over the pressure side of a Savonius style wind turbine (SSWT). Although, the wind energy market has been dominated by the horizontal axis wind turbines (HAWTs); recently, vertical axis wind turbines (VAWTs) are also finding emerging interest for off-grid applications $([1,2])$. As have been reported in the literature [3], the VAWTs possess a number of distinct advantages over HAWTs such as easy installation and maintenance, less installation space, self-starting at low wind speeds, wind acceptance from any direction, no need of yaw mechanism, easy fabrication, and less noise among others.

SSWT, as a member of VAWTs, appears to be promising for off-grid energy conversion because of its better self-starting capability and flexible design promises [4]. SSWT blades are characterized by relatively large surfaces, which are thin circular shape to produce large drag for power generation. Typically, the suction side of the advancing blade is submitted to strong adverse pressure gradient, causing a well known vortex shedding process, which is responsible for the wake flow. This topic has been the subject of many researches in the past decades, as it obviously depends on tip speed ratio (TSR) and directly influences the turbine efficiency $[5,6]$. The flow on the pressure side of the blade is generally considered as fully attached and is characterized by high pressure, low velocity level that produces most of the drag used in the energy conversion. However, because of the gap between the two blades, the flow is accelerated on the pressure side of the returning blade and a thicker boundary layer develops at this side. Because of the concave curvature of the blade and the small scale of the turbine, centrifugal 
instabilities (pairs of longitudinally elongated counter rotating vortices) may occur; and depending on the flow characteristics, can cause natural transition on the blade [7]. Moreover, these vortices induce different mechanisms of ejections and sweeps, causing thereby strong transverse variations of the drag coefficient, which results in the formation of hot spots near solid walls (i.e. blade surface) [8]. This can also lead to a rapid degradation of mechanical structures and materials fatigue. It has to be noted that because of the small scale of these turbines, viscous effects are of the primary importance in the prediction of the turbine performance, and it becomes necessary to lead detailed analysis of the flow physic to evaluate its influence.

In last few decades, a number of experimental and numerical investigations have been carried out on the performance analysis of SSWTs [3]. Several improvements had then been performed to increase their performances, this includes optimizing height to diameter ratio (aspect ratio, AR) [9], adding of end plates at the top and bottom of the turbine blades that marginally increases the performance [10], and several flow control systems [11]. Further, it is now usual to have a certain overlap between the turbine blades to accelerate the flow near the trailing edge of the advancing blade and insert pressure on the suction side of the returning blade [12]. This is often reported to be beneficial for the performance improvement of this turbine $[13,3]$. In [14], unsteady Reynolds-averaged Navier-Stokes (URANS) simulations were performed over a semi circular, SSWT. The authors investigated the gap flow, and concluded that a boundary layer flow occurs on the pressure side of the returning blade. This is due to acceleration and re-orientation of the flow generated by the gap (see Figure 1). The latter has a positive effect 
on the turbine performance, which produces more drag. However, because of the boundary layer development on the concave side of the blade surface (i.e. pressure side), the flow may become unsteady due to small scale disturbances. These flow features are not captured by URANS methods that are, however, still the most popular means to predict the turbine performances $[5,15]$.

The case of a boundary layer flow on a curved, circular wall, is typically associated with centrifugal instabilities. On such concave geometries, the wall-normal balance of forces on the fluid is modified by the presence of a centrifugal force, which increases away from the wall and is balanced by the pressure force. Next, a sharp wall-normal displacement of fluid in the boundary layer due to the surface roughness often destroys this balance and destabilize the shear layer. This instability mechanism may lead laminar boundary layer to transit to turbulence [16]. The phenomena of such centrifugal instability, known as Görtler instability, is relatively well studied, as numerous experimental, and more recently numerical studies have investigated this flow $[17,18]$. As observed in [18], the boundary layer instability appears with counter rotating steady vortices with constant span-wise wavelength, having a constant stream-wise location. As shown in [19] and [20], these vortices forms together with longitudinal streaks with low and high momentum because of the vertical displacement of the flow. These streaks then deform the flow and the low momentum regions form the well known mushroom like shapes structures [21]. Finally, it was also shown that the skin friction coefficient is increased compared to flow over a flat plate because nonlinear modification of the primary steady flow by wavy secondary 
instabilities [22].

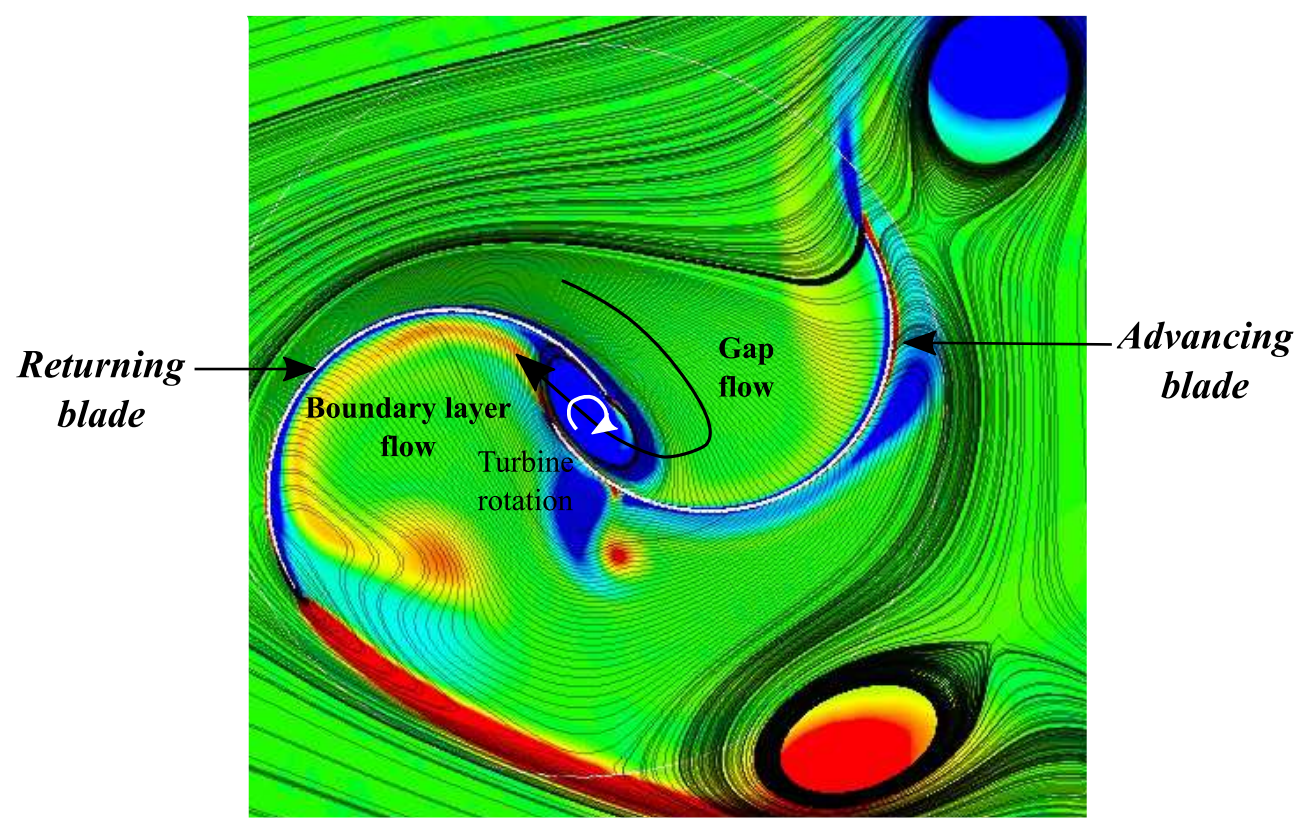

Figure 1: Vortex flow around a SSWT. URANS calculation from [14] at the tip speed rati of TSR $=0.8$, and Reynolds number of $R e=12.3 \times 10^{4}$

To demonstrate the presence of centrifugal instabilities on SSWTs, DNS computations are performed with the open-source spectral elements code NEK5000 [23] on the pressure side of a single blade, under a constant inflow condition with no turbine rotation. The main objectives of the present study are $(i)$ to verify if such flow can actually occurs on SSWT blades in the range of operating Reynolds number considered for this type of turbine $\left(1 \times 10^{5}<\right.$ $R e<2 \times 10^{5}$ ) (ii) to investigate the modification of boundary layer flow, and hence ( iii) the changes in aerodynamic performances, including friction, pressure and drag characteristics. 
The manuscript is organized as follow: after a presentation of the numerical method in section 2, a mesh sensitivity analysis is presented considering the element order with the same mesh. Since the present geometry (with high tip curvature) has never been investigated in the past, the spectral element method is validated with a curved wall of constant radius $R=1 \mathrm{~m}$ in section 3 , which is the most popular case to study centrifugal instabilities nowadays. A detailed analysis of the flow is then carried out in section 4. It is followed in the same section by the investigation of pressure, friction at the wall and drag and lift forces, which are compared with URANS simulations to highlight the contribution of the present work. Finally, the manuscript is concluded in section 5 .

\section{Numerical Model}

The dynamics of a three-dimensional incompressible flow of a Newtonian fluid are described by the Navier-Stokes equations

$$
\dot{\mathbf{U}}=-(\mathbf{U} \cdot \nabla) \mathbf{U}-\nabla P+R e^{-1} \Delta \mathbf{U},
$$

$$
\nabla \cdot \mathbf{U}=0 .
$$

Here $\mathbf{U}=\left(U_{x}, U_{y}, U_{z}\right)^{T}$ is the velocity vector, where $U_{x}, U_{y}$, and $U_{z}$ being its component along $x-, y-$, and $z$ - axes, respectively, and $P$ represents the pressure term. The velocity is non-dimensionalized by the free-stream velocity $U_{\infty}$ and the flow conditions are set according to the free-stream Reynolds number, $R e_{\xi}=U_{\infty} \xi / \nu$, where $\nu$ is the kinematic viscosity of the 
considered fluid and $\xi$ is the curvilinear distance between the leading edge and the training edge.

Navier-Stokes equations are solved using NEK5000 flow solver developed at Argonne National Laboratory [24]. It is based on the spectral elements method (SEM), introduced in [25], which provides spectral accuracy in space while allowing for the geometrical flexibility of finite element methods. Spatial discretization is obtained by decomposing the physical domain into spectral elements within which the velocity is defined on Gauss-Lobatto-Legendre (GLL) nodes and the pressure field on Gauss-Legendre (GL) nodes. The solution to the Navier-Stokes equations is then approximated within each element as a sum of Lagrange interpolants defined by an orthogonal basis of Legendre polynomials up to degree $N$. The results presented in this paper have been obtained with a target mesh that has a polynomial order of $N=8$. The total number of spectral elements in the mesh is $E=154 \times 10^{3}$. The convective terms are advanced in time using an extrapolation of order 3, whereas the viscous terms use a backward differentiation of the same order, resulting in the time-advancement scheme labeled BDF3/EXT3. NEK5000 employs MPI standards for parallelism [26, 27] and parallel computations have been performed on up to 4096 processors. For further details about the spectral elements method, the reader is referred to the books by [28] and [29].

The computational domain considered is shown in Figure 2. It is a C-type like mesh, having a total length of $7.08 c$ with $c=2 R$, where $R=1 \mathrm{~m}$ is the blade radius. It is noted that only the pressure side of the blade is considered in the current work and the turbine rotation is not considered. This is mainly for simplifying the geometry and to avoid the complex, multi scaled 


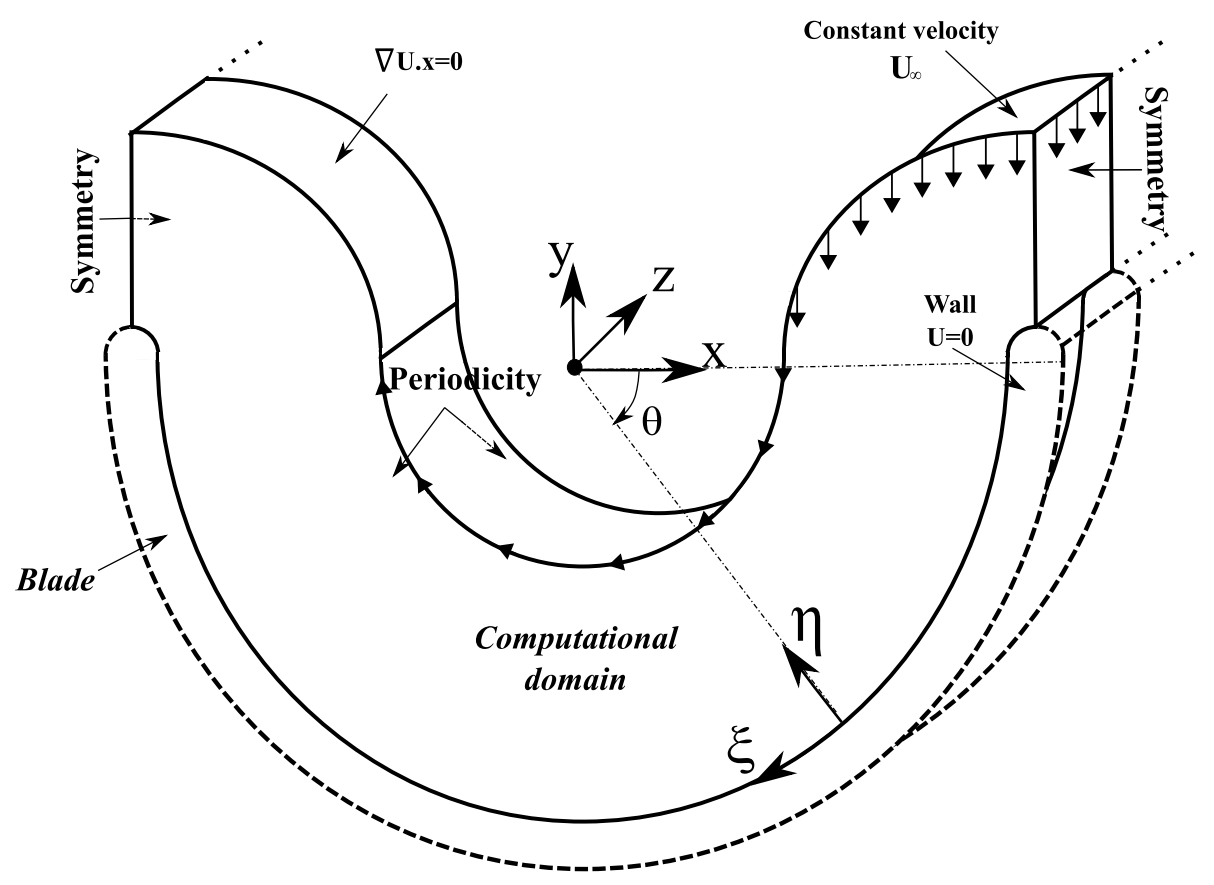

Figure 2: Computational domain and boundary conditions

simulation caused by adverse pressure gradient on its suction side that may also induce massive boundary layer stall. Considering similar studies, the domain is very large in the wall normal direction $(\eta=0.4 c)$ to capture the flow around the trailing edge, which can experience boundary layer detachments, and it is about 2 times longer in the tangential direction. The span has been set to $0.2 c$ to reduce computational cost. As shown in the result section, it is sufficient to capture enough characteristic wavelength and to avoid forcing the flow in the span-wise direction [30].

A velocity condition is set at the inlet and for the inner circle. It is given by $U_{\infty}=\bar{U}+u^{\prime}$, where $\bar{U}$ is the magnitude of the mean velocity chosen in order to match the previous study shown in Figure 1 (i.e. $R e=U_{\infty} D / \nu=12.3 \times 10^{4}$ ) with $D=2 c$ being the turbine diameter. As only one blade is considered, the 
characteristic length is the curvilinear distance between the leading edge and the trailing edge of the blade. The Reynolds number for the current study is then $R e_{\xi}=9 \times 10^{4}$. Furthermore, $u$ is a velocity perturbation, which set a turbulence intensity of $1 \%$ at the inlet. It is applied in the code using random number generation in each cell, leading to the frozen isotropic turbulence of length scale equal to the cell size at the inlet i.e. $\lambda_{t}=0.02 m$, whereas no time correlation is applied. The outlet boundary condition is set as $\nabla \mathbf{U} \cdot \mathbf{x}=0$. In order to reduce the CPU time, periodic boundary conditions are imposed on the vertical side planes of the domain (span-wise direction); whereas a no slip condition is set on the blade surface.

Six sets of 37 monitor points at the wall surface are set to record the wall pressure and friction velocities along the chord. It is equally distributed along the chord, starting right downstream the leading edge $\left(\theta=0^{\circ}\right)$ with a step of $\Delta \theta=5^{\circ}$, and located respectively at $z=0.03 \mathrm{~m}, z=0.06 \mathrm{~m}, z=0.09 \mathrm{~m}$, $z=0.12 \mathrm{~m}$ and $z=0.18 \mathrm{~m}$ to capture the spanwise variation of the flow.

As shown in Figure 3, mesh refinements are used at the leading edge, the trailing edge, and in the near wake region. A total number of 385 spectral elements are set along the chord, whereas the wall normal direction is discretized with 40 spectral elements and the spanwise direction is composed of 10 elements. It results in a total number of $E=154 \times 10^{3}$ spectral elements.

To verify the mesh convergence, four different element orders are tested, as depicted in Figure 4. The calculations are first converged in time and then run for a short time to obtain the averaged values of the forces. The 


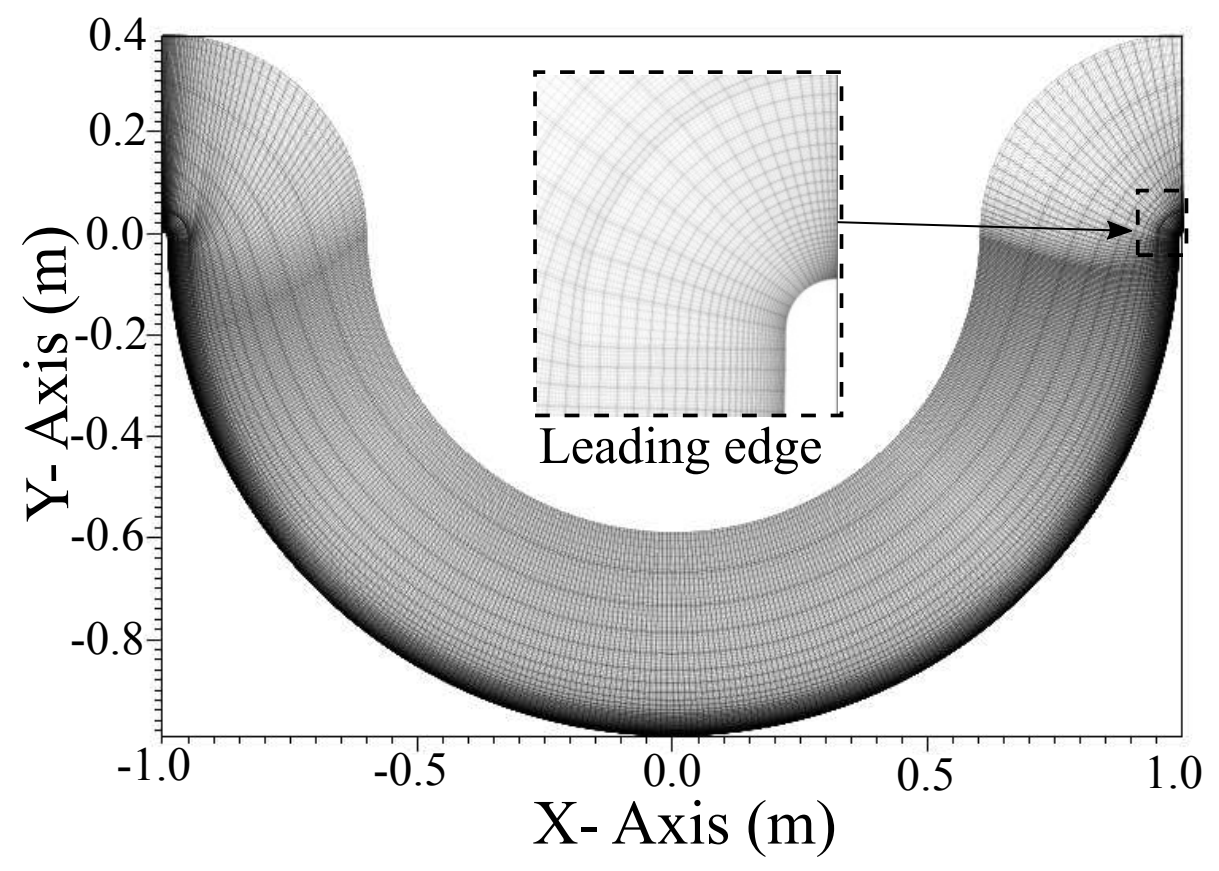

Figure 3: Mesh, $E=154 \times 10^{3}$ spectral elements, $N=8$

lowest order mesh does not permit to capture the Görtler modes and is not sufficient to ensure a DNS accuracy. A relatively low order mesh $N=$ 6 ensures DNS calculation in most of the domain, however the wake and the spanwise wavelength of Görtler modes are not correctly described. The order mesh $N=8$ is then necessary to get a full DNS resolution in all the fluid domain. Finally, increasing the mesh order to $N=10$ is not affecting the simulation results. In order to confirm these results quantitatively, the convergence of the pressure and the viscous components of the lift and drag forces are presented in Table 1 . The viscous components of the lift $\left(C l_{v}\right)$ and drag $\left(C d_{v}\right)$ converge for $N=6$, whereas it is necessary to use $N=8$ to obtain a good convergence on their pressure counterparts $\left(C l_{p}\right.$ and $\left.C d_{p}\right)$, 
and hence the total lift and drag $\left(C l_{t}\right.$ and $\left.C d_{t}\right)$. This confirms that $N=$ 8 is necessary to obtain full DNS resolution in the whole computational domain. Therefore, considering the tradeoff between computational costs and numerical accuracy, the latter is chosen for the rest of the paper, which has a final number of $112 \times 10^{6}$ points, leading to $\Delta \xi / \Delta z \approx 0.5$ along $80 \%$ of the wing surface. The boundary-layer mesh is kept constant and the first cell size has been set in order to obtain $\eta_{+}=\eta u_{\tau} / \nu<0.2$ along the chord, where the $u_{\tau}$ is the friction velocity. This will also allow us to investigate higher Reynolds numbers in the future. Therefore, the main mesh parameters for the DNS calculation are $\eta_{+, \text {min }}<0.2, \xi_{+, \text {min }} \approx 1$ and $z_{+, \text {min }} \approx 2$ at the blade surface.

The calculation is first initialized with the low order mesh at $N=6$ in order to save computational time. After its convergence, the solution is taken as the initial condition for the higher order mesh at $N=8$, which in turn is converged in time after what the calculation is performed the time needed for the post processing.

As shown in Figure 5, the flow instability and the transition to turbulence is triggered by the blade leading edge, which has a relatively high curvature, together with the random perturbation at the inlet. This induces a laminar boundary layer detachment right after the leading edge, and a laminar reattachment at $\theta=6.3^{\circ}$. This region is quasi-steady and has small fluctuations that destabilize the flow downstream. The length scale is then fixed to the surface element size. This is taken as a first step for the present study, however, a receptivity analysis will be performed in the future, based on the influence of free stream turbulence. 


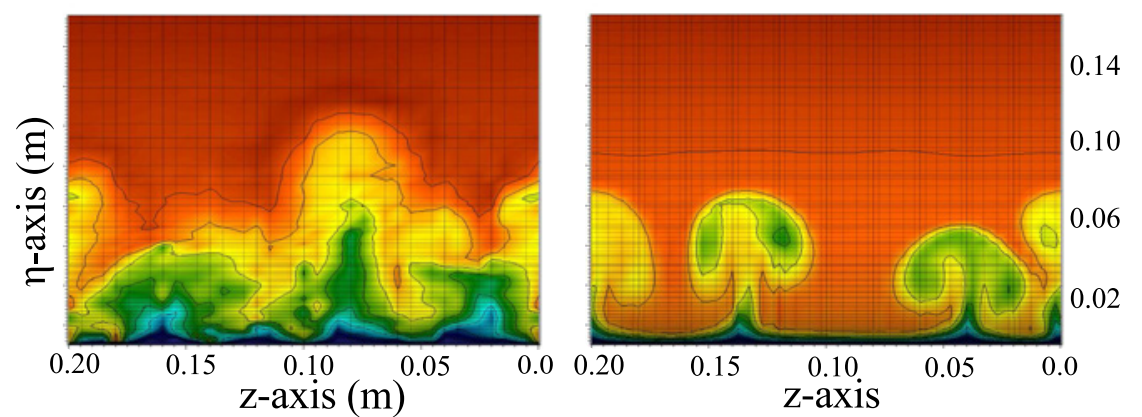

(a) $\mathrm{N}=4$

(b) $\mathrm{N}=6$

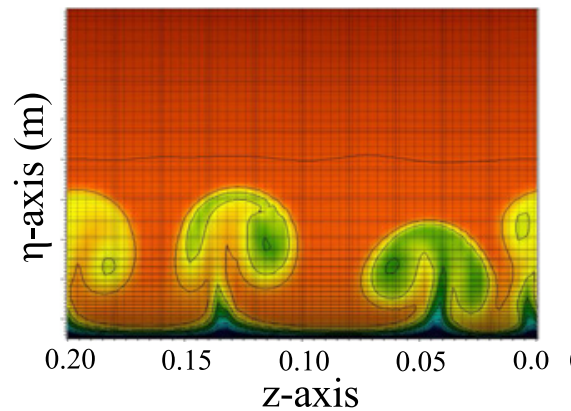

(c) $\mathrm{N}=8$

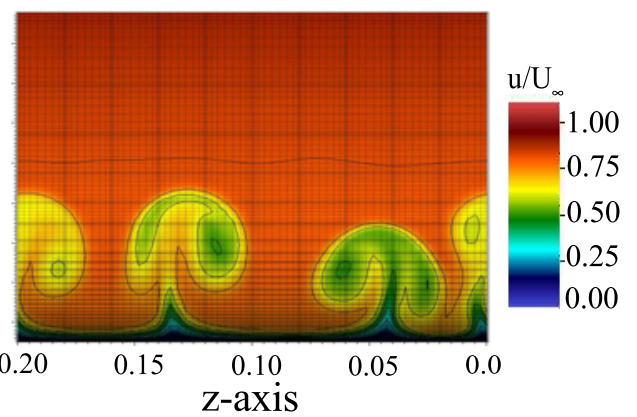

(d) $\mathrm{N}=10$

Figure 4: Development of Görtler vortices as a function of element order $N$

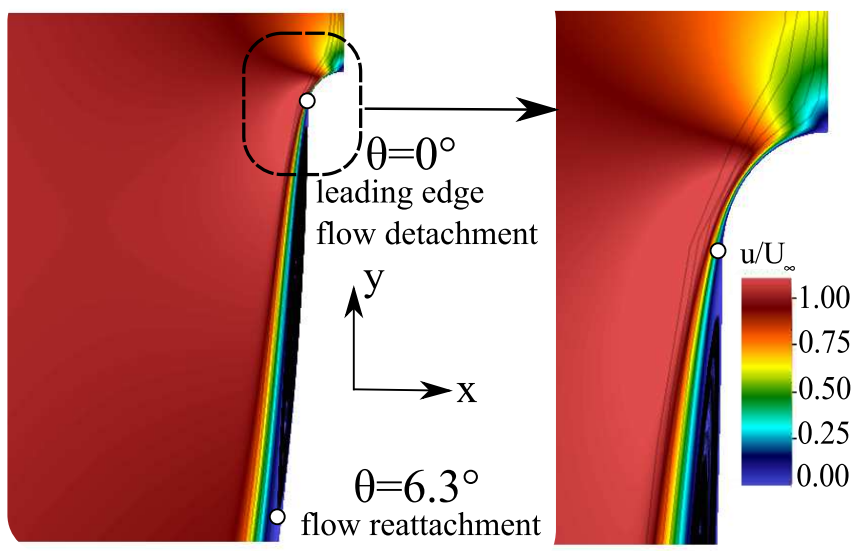

Figure 5: Reversed flow downstream the blade leading edge 


\begin{tabular}{lccc}
\hline & pressure drag $C d_{p}$ & viscous drag $C d_{v}$ & total drag $C d_{\text {tot }}$ \\
\hline$N=10$ & 0.5395 & -0.0021 & 0.5416 \\
\hline$N=8$ & 0.5392 & -0.0019 & 0.5373 \\
\hline$N=6$ & 0.5284 & -0.0019 & 0.5266 \\
\hline$N=4$ (not converged) & 0.5739 & -0.0012 & 0.5727 \\
\hline \hline & pressure lift $C l_{p}$ & viscous lift $C l_{v}$ & total lift $C l_{\text {tot }}$ \\
\hline$N=10$ & 0.0190 & -0.0078 & 0.0113 \\
\hline$N=8$ & 0.0194 & -0.0077 & 0.0117 \\
\hline$N=6$ & 0.0147 & -0.0076 & 0.0071 \\
\hline$N=4$ (not converged) & 0.0349 & -0.0080 & 0.0269 \\
\hline
\end{tabular}

Table 1: Comparison lift and drag coefficients for different spectral element order N.

\section{Validation study}

The Savonius blade calculation is first validated on a simple concave curved wall, where a reference study based on an equivalent DNS using finite volume algorithm is available in the literature [16]. The goal is to show the ability of the current method to capture the centrifugal instabilities. Therefore, only in this section, a domain size with a spectral element mesh identical to [16] is considered and is shown in Figure 6. The quarter of a circle with the radius of $R=1 \mathrm{~m}$ and thickness of $\eta=0.1 \mathrm{~m}$ is defined as in [16]. This lead to $\xi_{+}=1500$ and $\eta_{+}=100$ for the non dimensional stream-wise and wall normal directions. To optimize the computational time, the span is reduced to $L_{z}=0.05 \mathrm{~m}$ compared to $L_{z}=0.0892$ in [16]. This is found to be enough to capture at least 5 mushroom structures in the span-wise direction, compared to 9 in the reference study. The number of spectral element is $N_{\xi}=120, N_{\eta}=20$ and $N_{z}=10$ with order 8 , leading to a total of $12 \times 10^{6}$ 
points, compared to $17 \times 10^{6}$ in the reference study due to a smaller span of the present domain. The details of the mesh parameters are given in Table 2 . A Blasius profile with an external velocity of $U_{\infty}=2.8 \mathrm{~m} / \mathrm{s}$ and a thickness of $\delta=0.00373 \mathrm{~m}$ is defined at the inlet. A random perturbation equivalent to the blade calculation is also defined with a turbulence intensity of about $1 \%$. $U_{\infty}=2.8 \mathrm{~m} / \mathrm{s}$ is also set at the upper wall, leading to a maximum Reynolds number of $R e_{\xi}=28.2 \times 10^{4}$, which induces laminar to turbulent transition and fully turbulent flow over more than $60 \%$ of the domain. Other boundary conditions are identical to those presented in section 2.

The space and time averaged wall friction coefficient is first compared with

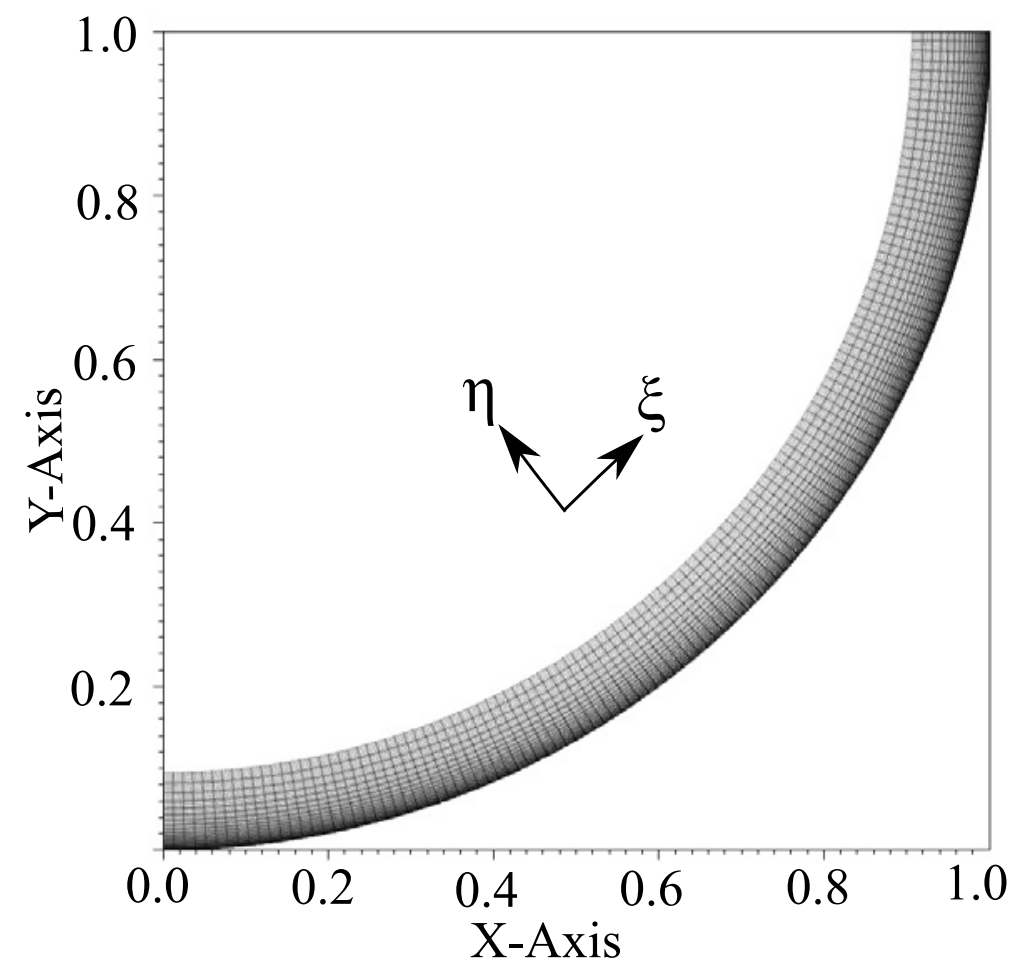

Figure 6: Spectral element mesh for the curved wall, number of element $E=24 \times 10^{3}$ 
the DNS of [16] and the experiments of [31]. In addition, the laminar and turbulent solutions for a flat plate are shown. The wall friction inside the laminar region compares well with the previous DNS and the experiment. The level of friction follows the laminar flat plate solution, as there is no development of centrifugal instability in this region. The friction coefficient increases earlier in the measurement of [31] compared to both DNS simulations. This is due to the experimental set up at the inlet of the test section that consider series of 12 perturbation wires with constant spacing. This tends to force the inception of centrifugal instabilities, which causes early transition in the boundary layer. It can be seen than both DNS capture the transition at the same location, and the level of wall friction inside the turbulent boundary layer agree well, where higher values are obtained compared to the turbulent flat plate solution.

The development of Görtler instability is shown in Figure 8. The same boundary layer thickness is observed in Figure 8(a), and the inception of longitudinal streaks is equivalent to that of [16]. Because the inlet perturbation is different between the two computations, the streaks are organized slightly differently, however, the overall wall normal development and spanwise wavelength match very well. In particular, the distinct behavior between isolated mushrooms and merging mushrooms developments are reproduced by the present DNS.

The evolution of the local Görtler number, represent the ratio between destabilizing centrifugal to stabilizing viscous forces, and two boundary layer velocity profiles are then compared in Figure 9. The Görtler number is based on the boundary layer momentum thickness $\theta_{t}$, the radius $R$, the upstream 


\begin{tabular}{lcccc}
\hline & $\operatorname{span} L_{z}(\mathrm{~m})$ & $N_{\xi}-N_{\eta^{-}} N_{z}$ & total grid points & $\Delta_{\xi}^{+}-\Delta_{\eta}^{+}-\Delta_{z}^{+}$ \\
\hline present DNS & 0.05 & $960-160-80$ & $12 \times 10^{6}$ & $16.8-0.56-6.42$ \\
\hline Schrader et al. (2011) & 0.0892 & $1025-129-129$ & $17 \times 10^{6}$ & $15.7-0.7-7.1$ \\
\hline
\end{tabular}

Table 2: comparison of mesh resolution, present DNS vs DNS of Schrader et al. (2011)

velocity $U_{\infty}$ and the kinematic viscosity $\nu$, and is defined as:

$$
G_{\theta_{t}}=\frac{U_{\infty} \theta_{t}}{\nu} \sqrt{\frac{\theta_{t}}{R}} .
$$

In Figure 9 (a), both DNS first match with the laminar flat plate solution. Around $\xi_{+}=400, G_{\theta}=8$, the inception of longitudinal streaks increases the Görtler number due to an increase of the momentum thickness; both DNS match very well and go away from the laminar flat plate solution. Finally, the Görtler number of the present DNS is lower that the DNS of [16] from the breakdown region (around $\theta=35^{\circ}$ ), and keep lower values until the end of the fluid domain. This difference can be attributed to (i) differences in the space-time averaging due to development of highly three dimensional flow, and (ii) because of the influence of inlet perturbation that modifies the breakdown of streaks.

Two boundary layer velocity profiles are also compared in Figure 9 (b). At $\theta=33.7^{\circ}$, the flow is governed by the Görtler streaks and both velocity profiles are highly inflectional, which is captured by the present DNS. Downstream, the boundary layer is fully turbulent, and a minor difference is observed between the two computations. 


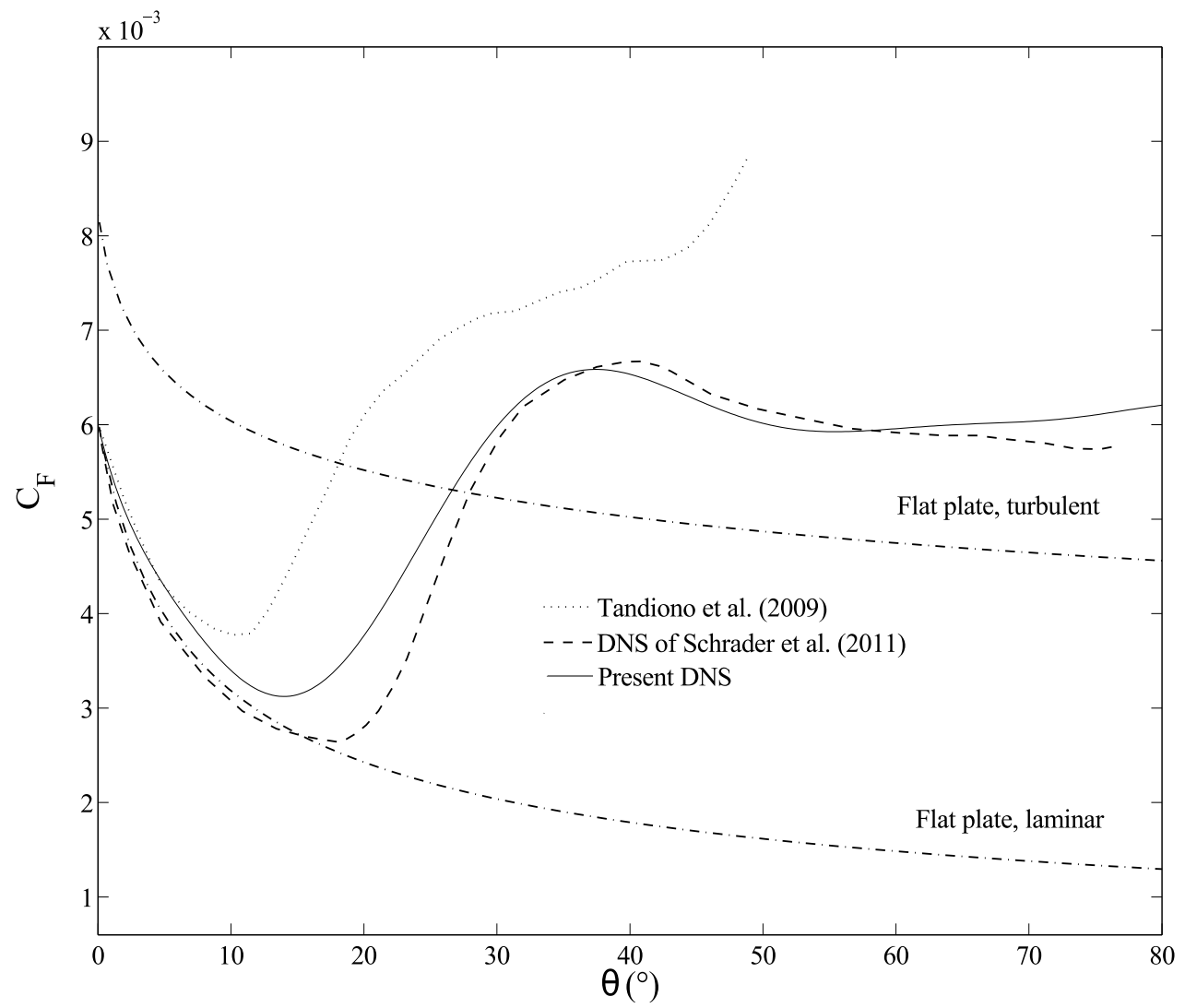

Figure 7: Comparison of the spanwise and time averaged wall friction coefficient along the curved plate; present DNS vs DNS of Schrader et al. (2011) and experiments of Tandiono et al. (2009)

\section{Results and discussions}

The iso surface of $\lambda_{2}$ criterion colored with contour of velocity is shown in 


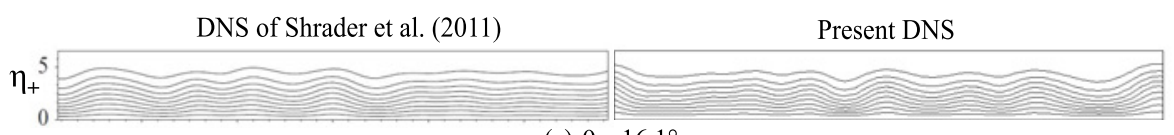

(a) $\theta=16.1^{\circ}$

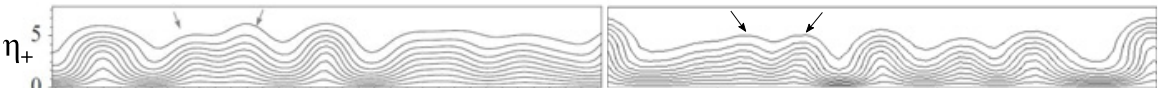

(b) $\theta=21.6^{\circ}$

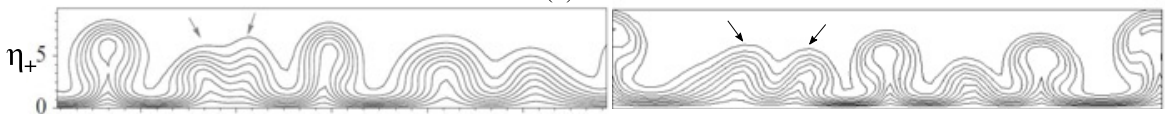

(c) $\theta=27.1^{\circ}$

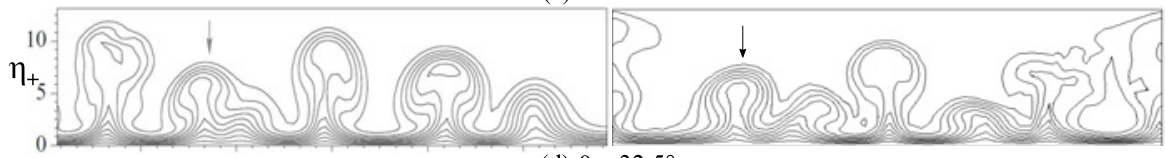

(d) $\theta=32.5^{\circ}$

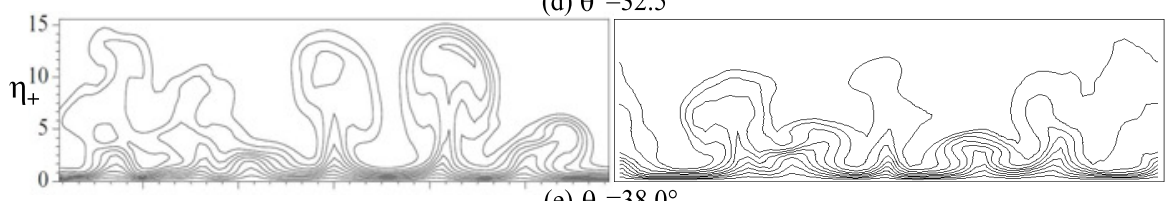

(e) $\theta=38.0^{\circ}$

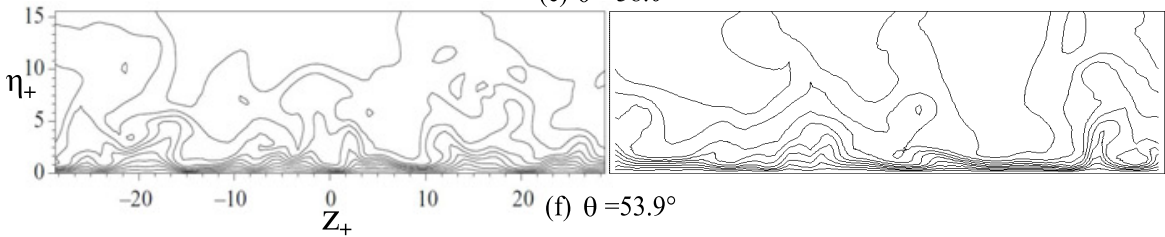

Figure 8: Development of Görtler instability. Velocity profiles for different angles $\theta$; present DNS vs DNS of Schrader et al. (2011)

characterized by the appearances of typical horseshoe vortical structures.

The flow then extend in the wall normal direction and smaller scales vortices are observed, which are identified as sinuous streaks. These streaks are observed up to the transition process, around $\theta=170^{\circ}$, where the flow becomes fully turbulent.

Figure 11 shows the instantaneous evolution of boundary layer flow and the corresponding boundary layer thickness. It is taken at $z=0.06$, where a classical mushroom structure develops. A laminar boundary layer develops 


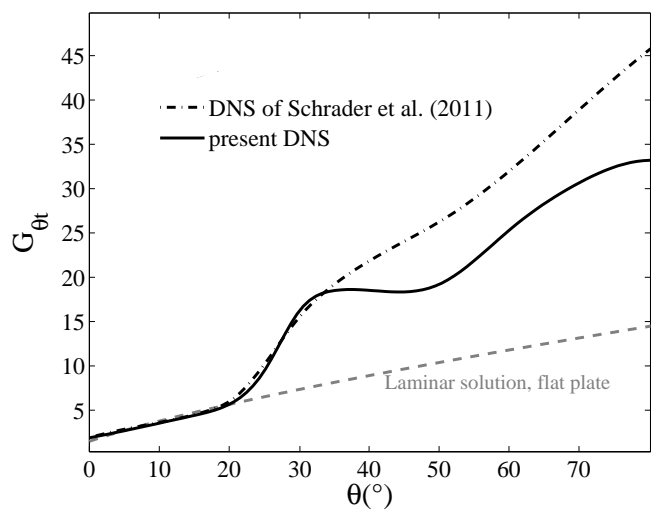

(a)

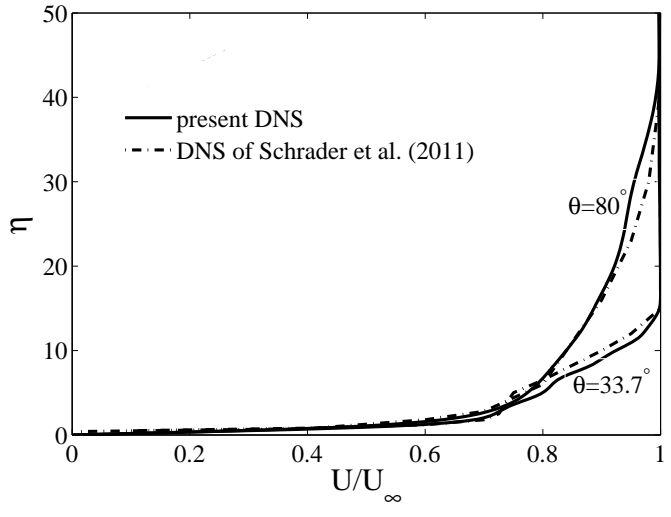

(b)

Figure 9: Validation of (a) Local Görtler number and (b) Mean velocity profiles (temporal spanwise average).

from the leading edge of the blade, and reaches to its maximum thickness before the inception of Görtler vortices. When streaks appear, the boundary layer thickness starts to stagnate (Figure 11 (b)), whereas the low speed region suddenly increases due to the presence of streaks (Figure 11 (a)). With the development of secondary instabilities, periodic structures are observed in the stream-wise direction, until the flow transition to turbulence near the trailing edge, highlighted by higher velocities.

To demonstrate that the centrifugal instabilities are correctly captured, the evolution of Görtler number is shown in Figure 12. At first, the present DNS calculation match the typical values for a laminar boundary layer (where $\left.\theta_{t}=0.664 / \sqrt{R e}\right)$. A sudden increase is observed around $G_{\theta_{t}}=8$ at $\theta=40^{\circ}$, which is the typical value where the Görtler vortices appear, also observed for example in [18] and [16]. The DNS solution doesn't match anymore with the laminar solution for a flat plate, since the flow becomes three-dimensional 


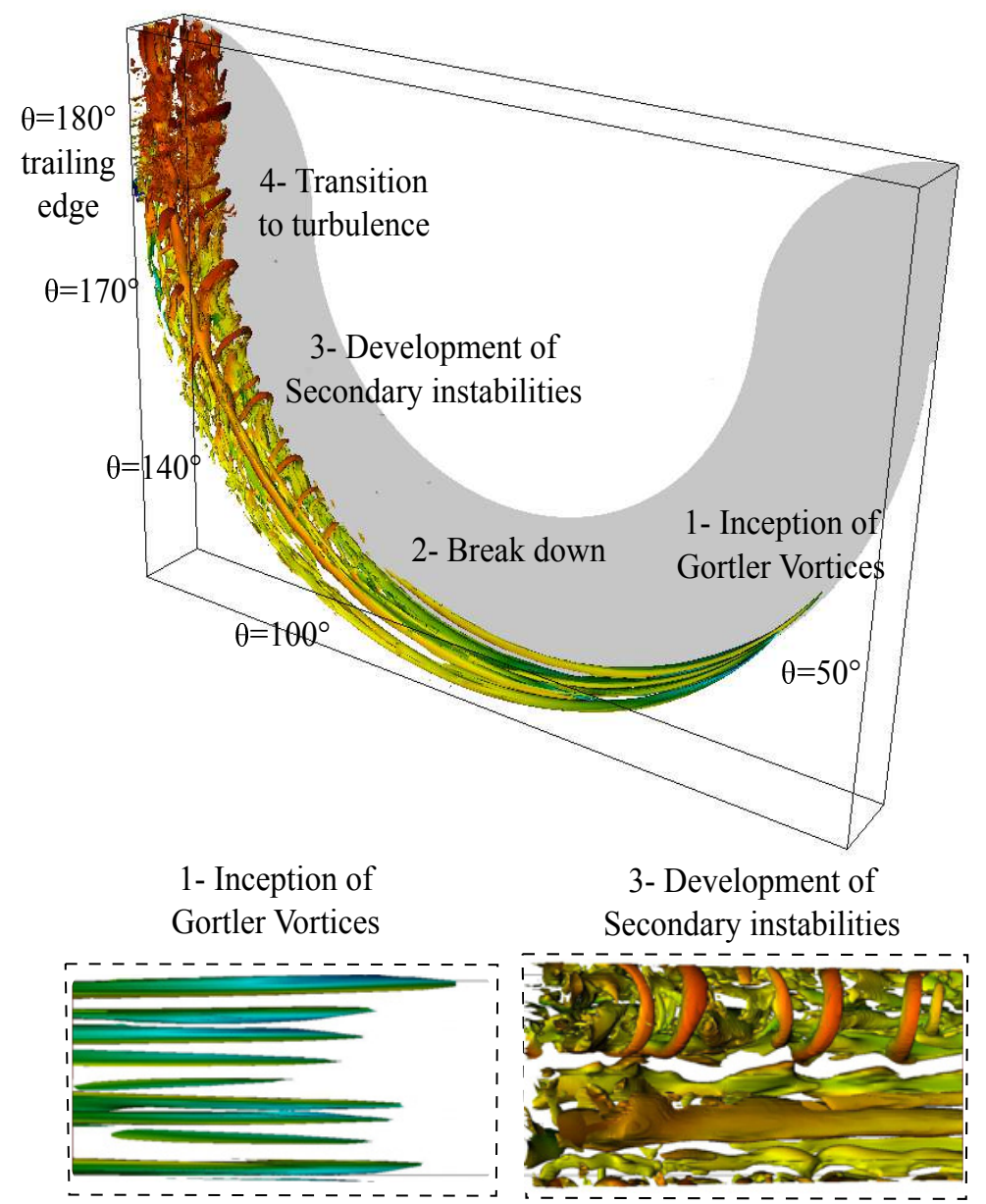

Figure 10: Coherent structure at the blade surface, iso surfaces of $\lambda_{2}$

where a progressive transition to turbulence occurs.

Figure 13 shows the variation of the velocity magnitude in the $(z, \eta)$ plane. Streaks are seen in the first stage of the physical mechanism, with the wavelength enforced by the inflow conditions. The first span-wise modulation is observed at $\theta=30^{\circ}$ (Figure 13 (a)). The streaks then develop in the wall normal direction and form low speed streaks (Figure 13 (b)) that progres- 


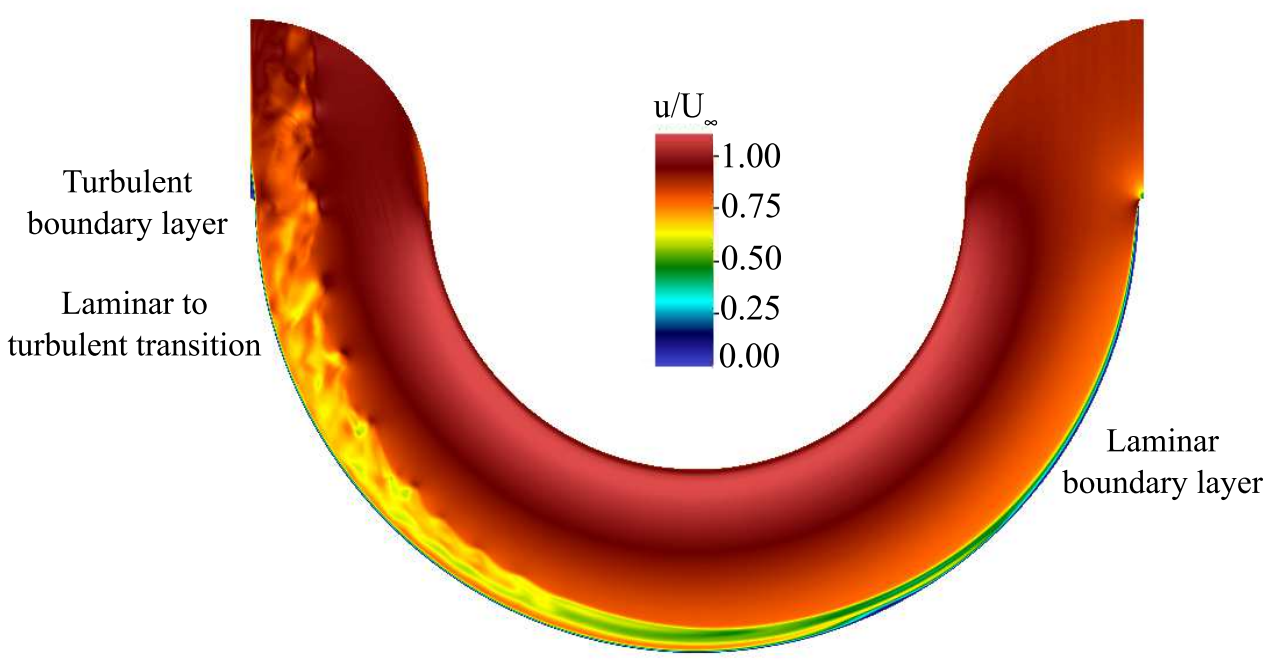

(a)

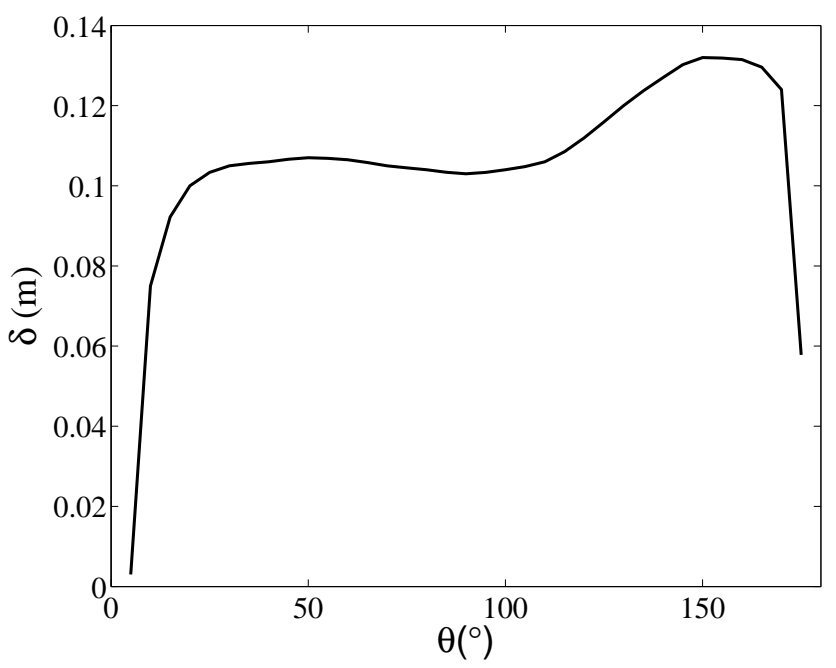

(b)

Figure 11: (a) Velocity field in the $(\xi, \eta)$ plane, (b) boundary layer thickness, $z=0.06 \mathrm{~m}$ 


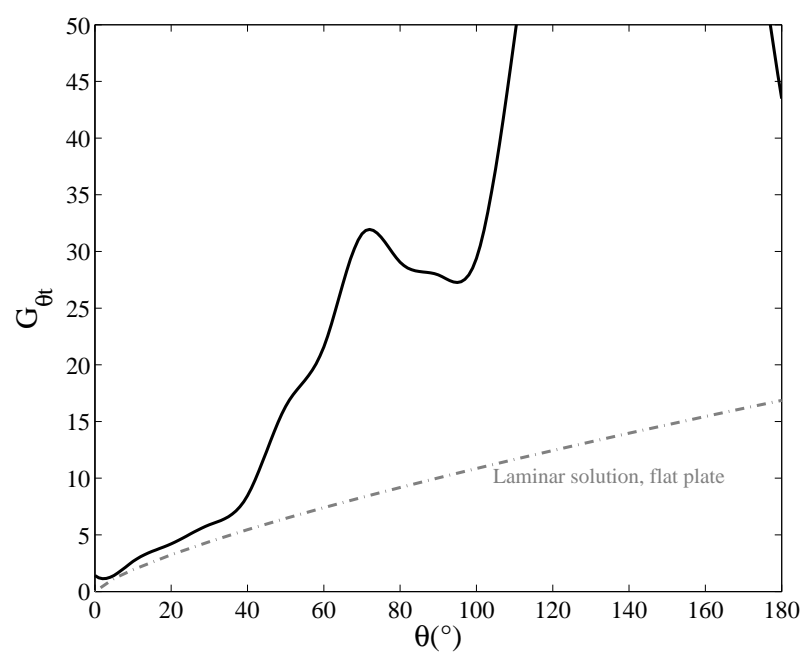

Figure 12: Variation of the Görtler number along the blade chord

sively form mushroom structures (Figure 13 (c)). Since the wavelength is relatively small compared to the mushroom overall size, the structures compete with each other. As a consequence, the structures close to each other are merging whereas they continue to develop in the wall normal direction (Figure 13 (b) to (d)). At this stage, the wavelength reaches a periodic state, which are directly related to the Reynolds number. In Figures 13 (e) to (g) two distinct behavior are observed. The right side of the domain shows a characteristic mushroom development, where the upper structure moves in the wall normal direction and detaches, after what multi-scale structures are observed. The left side mushroom competes again with another mushroom structure and merges during the development, which keep larger coherent structures and maintain the laminar flow. This is also highlighted by Figure 10. This behavior seems consistent with the validation case, where faster transition to turbulence was observed with less variations of the physic in the 


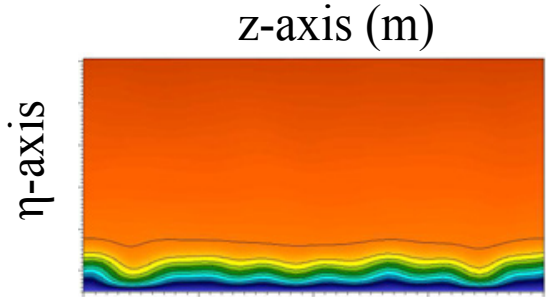

(a) $\theta=30^{\circ}$

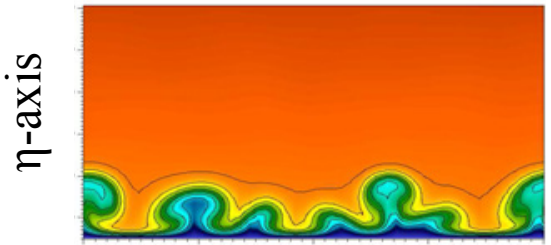

(b) $\theta=50^{\circ}$

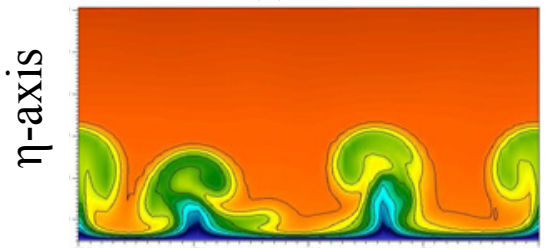

(c) $\theta=70^{\circ}$

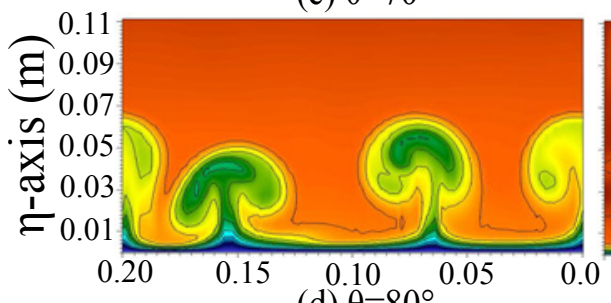

(d) $\theta=80^{\circ}$ z-axis

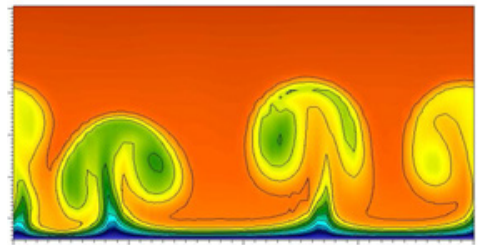

(e) $\theta=90^{\circ}$

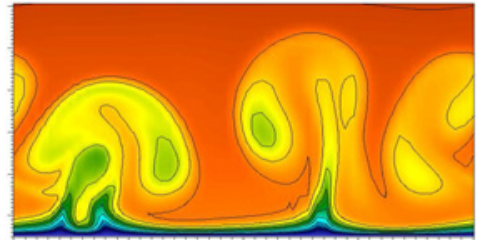

(f) $\theta=110^{\circ}$

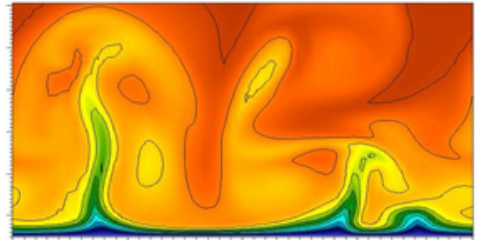

(g) $\theta=140^{\circ}$

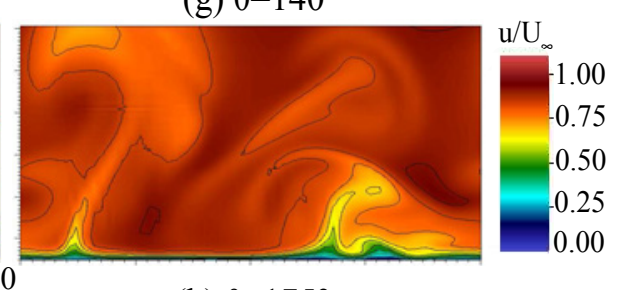

(h) $\theta=175^{\circ}$

Figure 13: Contour of velocity magnitude in the $(z, \eta)$ plane

To get a full observation on the development of Görtler flow throughout the domain, slices in the $(\xi, \eta)$ plane are shown in Figure 14. Near the wall 

observed.

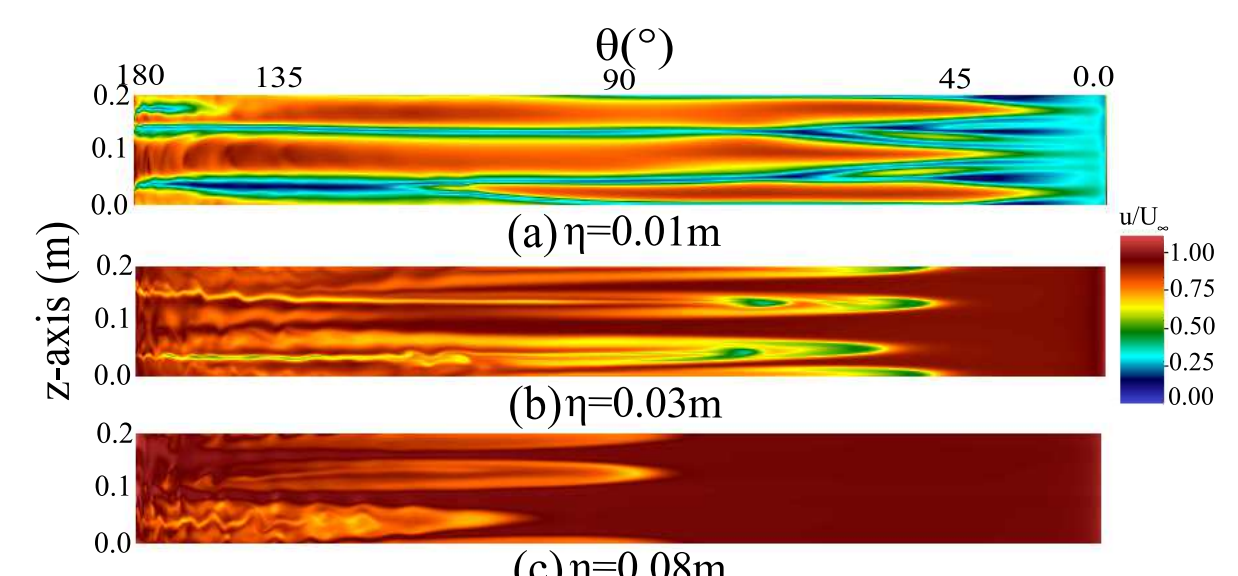

(c) $\eta=0.08 \mathrm{~m}$

(Figure $14(\mathrm{a})$ ), the low velocity field illustrates the merging of longitudinal streaks formed by the inlet perturbation after $\theta=40^{\circ}$. A second merging occurs from $\theta=45^{\circ}$ and creates a large laminar streak. It can be seen in this contour plot that the Görtler flow is mainly directed in the streamwise direction. In Figure 14 (b), inception of mushroom like structures is visible, highlighted by the green velocity field. First spanwise disturbances are observed around $\theta=110^{\circ}$, which is the beginning of the transition process. Near the trailing edge, the Görtler flow develop in the wall normal and spanwise directions with high velocity field (Figure $14(\mathrm{c})$ ), where turbulence is

Figure 14: Contour of velocity magnitude in the $(\xi, \eta)$ plane

The stream-wise skin friction coefficient, $C_{F \xi}=\tau_{\xi} /\left(0.5 \rho U_{\infty}^{2}\right)$, as function of $R e_{\xi}$ is shown in Figure 15 for three different $z$ locations, and for the span-wise averaged case. Here $\tau_{\xi}$ is the wall shear stress caused by flow in stream-wise direction and is calculated from the monitoring points placed at the cells adjunct to the wall. After obtaining the convergence, each point 
is time averaged over the whole simulation period. This Figure shows the resulting mean value in continuous black line, as well as the minimum and maximum values in dotted lines, which highlights the fluctuations. It is also compared with the flat plate solution, for both laminar and turbulent boundary layer flows. First, Figure15 (a) shows the evolution of the wall friction at the location where the mushroom-type structure develops and breakdown to turbulence occurs (i.e. $z=0.06 \mathrm{~m}$ ). Because of the small reversed flow area downstream of the leading edge, negative value of the skin friction coefficient is observed. The behavior is fully steady, and it reaches the friction level close to the laminar boundary layer around $R e_{\xi}=2 \times 10^{4}$, i.e. $\theta=20^{\circ}$. When the Görtler vortices appear at $R e_{\xi}=2 \times 10^{4}$ or equivalently as $\theta=40^{\circ}$, the friction coefficient suddenly increases and stays steady. Fluctuations appear in the breakdown region (around $R e_{\xi}=5 \times 10^{4}$ ), and increase up to the transition to turbulence near the trailing edge, where the friction reaches a level close to the turbulent boundary layer for the flat plate.

Figures 15 (b) and (c) show different behaviors. At these locations (i.e. $z=0.012 \mathrm{~m}$ and $z=0.018 \mathrm{~m})$, there is competition and merging of two mushroom structures, which largely increases the wall friction (see in the range $\left.20 \times 10^{4}<R e_{\xi}<5.5 \times 10^{4}\right)$. Since the coherent structures are larger up to the trailing edge, the level of friction is higher in the transitional region. Finally, the span-wise averaged friction coefficient in Figure 15 (d) appears to be higher than the typical value for a turbulent boundary layer. The reasons are the development of Görtler flow, combined with mushroom merging that increases the wall friction.

To highlight the development of three-dimensional flow together with sec- 


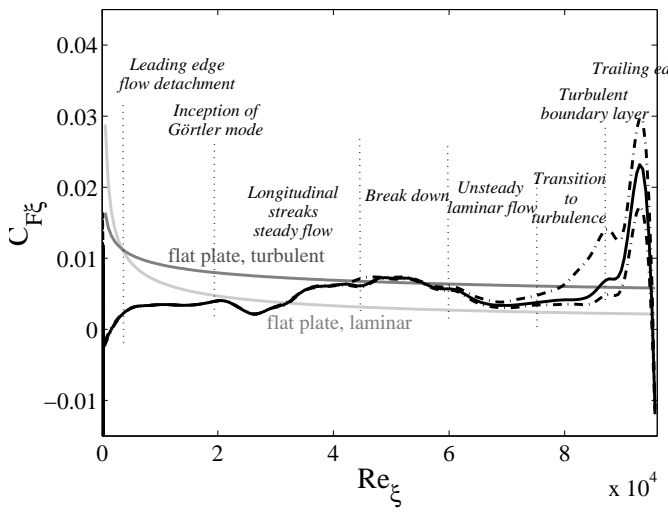

(a) $z=0.06$

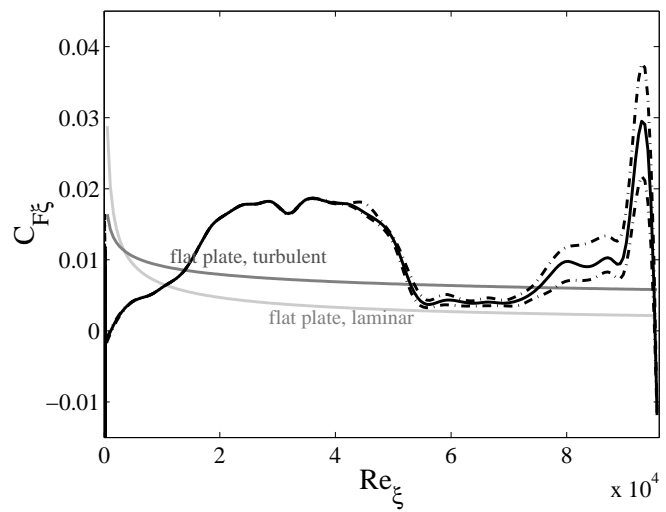

(c) $z=0.18$

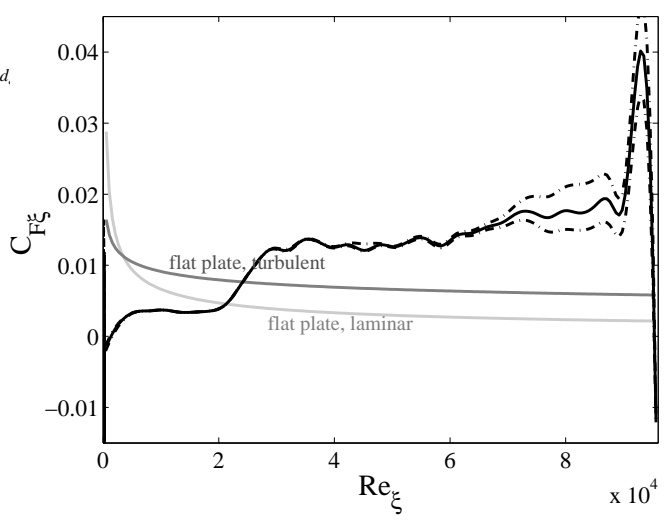

(b) $z=0.12$

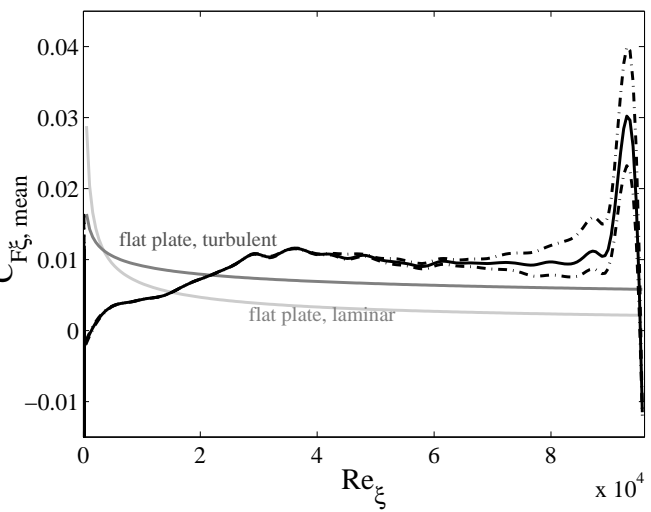

(d) spanwise averaged

Figure 15: Time averaged stream-wise friction coefficient along the blade chord. (-) mean values, (-.) fluctuations. 
ondary instabilities, the time averaged skin friction coefficients in the spanwise direction $C_{F z}=\tau_{z} /\left(0.5 \rho U_{\infty}^{2}\right)$ are plotted in Figures 16 (a) to (c) for the three span locations, and for the span-wise averaged case in Figures 16 (d). Here $\tau_{z}$ is the wall shear stress caused by flow in the span-wise direction. Large span-wise fluctuations are clearly observed from the inception of Görtler mode, which are representative of development and breakdown of longitudinal streaks, and the establishment of turbulent flow. The results highlight fluctuations from the breakdown region, where the flow becomes three dimensionnal around $\theta=120^{\circ}$. Larger fluctuations are observed after the breakdown of the longitudinal streaks, where the flow becomes highly unsteady and three dimensional (Figure 16(a) between approximately $\left.120^{\circ}<\theta<180^{\circ}\right)$. The spatially averaged value in Figure 16 (d) is close to zero with symmetric fluctuations, however, more points in the span-wise direction would be necessary to reach a complete balance (i.e. net 0 value).

Details of horseshoe like structures are shown in Figure 17, where the iso $\lambda_{2}$ with contour of the stream-wise vorticity are plotted in the unsteady region of $120^{\circ}<\theta<160^{\circ}$. Opposite vorticity levels are observed on the two sides the sinuous streaks in the span-wise direction, which clearly help for the development and stretching of mushroom structures along the $\eta$ axis, until their detachment. This behavior is initiated by a pair of contra rotative vortices that take place in the core of the mushroom structure and help the inception of secondary instability. When the structure develops, vorticities progressively mix together and smaller structures appear in the core, which is the first stage of transition to turbulence. It then produces the breakdown 


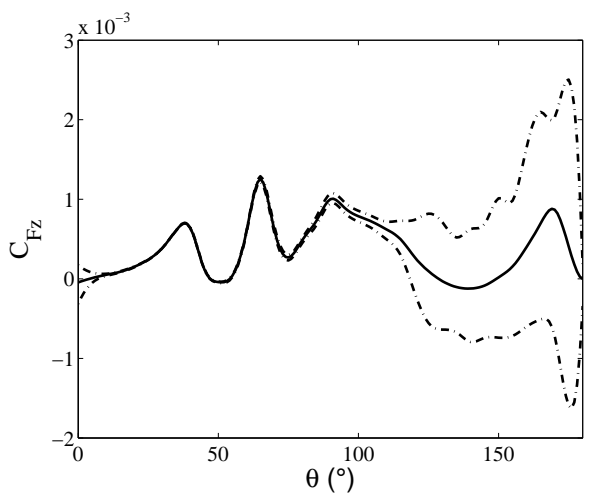

(a) $z=0.06$

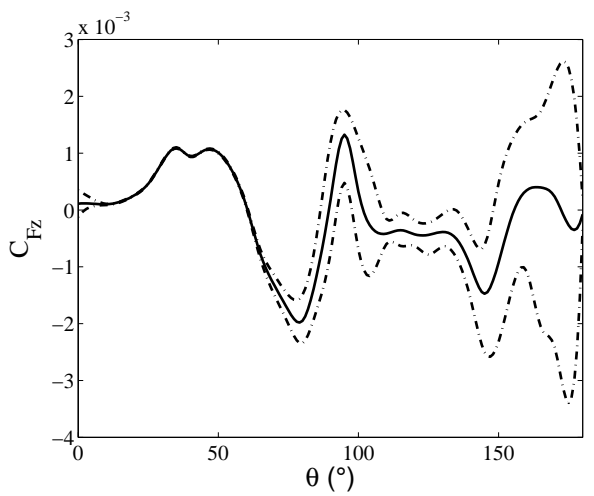

(c) $z=0.18$

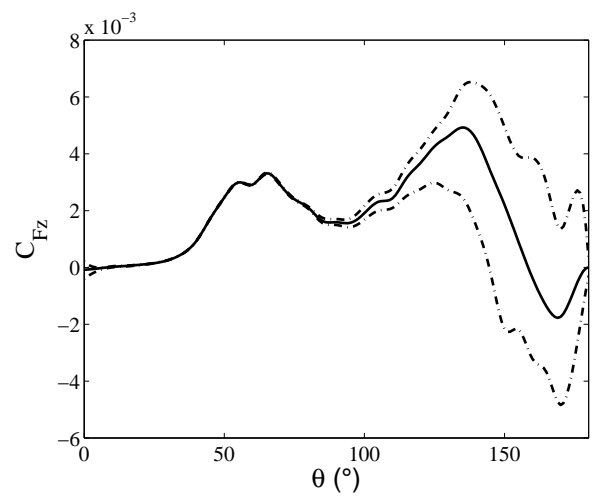

(b) $z=0.12$

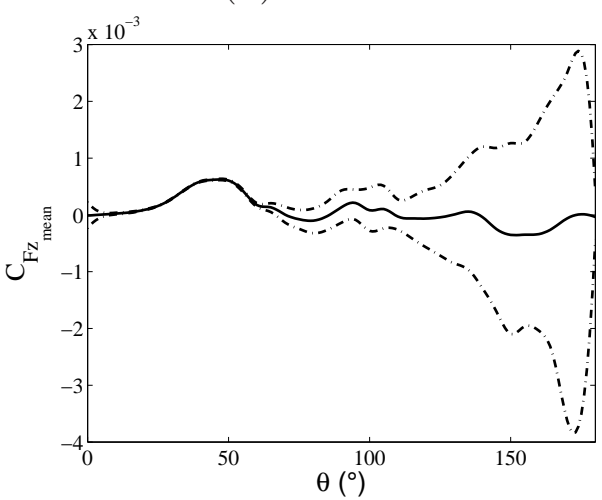

(d) spanwise averaged

Figure 16: Time averaged spanwise friction coefficient along the blade chord. (-) mean values, (-.) fluctuations. 
of secondary structures and hence laminar to turbulent transition occurs.

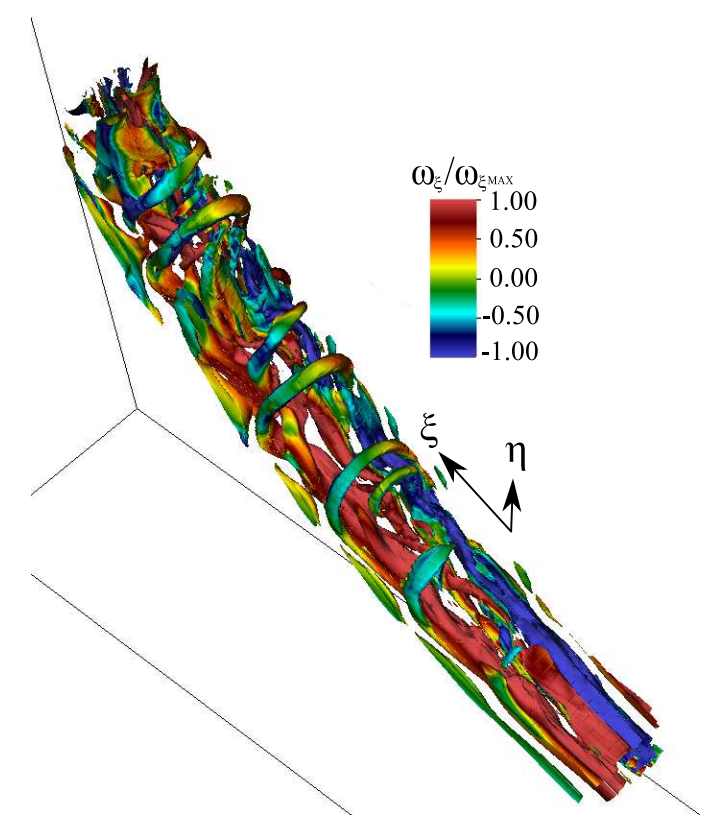

Figure 17: Detail of secondary instability, iso $\lambda_{2}$ and streamwise vorticity contour plots

To demonstrate the effect of centrifugal instabilities and the modification of the boundary layer regime caused by laminar to turbulent transition, on the blade performance, the DNS results are compared with three-dimensional URANS computation performed with the commercial software STARCCM+. The exact same computational domain is defined in the URANS solver. This allows to directly quantify the influence of centrifugal instabilities on the boundary layer flow and forces on the blade. It is discretized with unstructured polyhedral, whereas a structured mesh is defined in the boundary layer region with low Reynolds resolution, leading to $\eta_{+}=1$. The total number of cells are $N=839 \times 10^{3}$, which is found to be sufficient to fully solve for the boundary layer flow. A detailed space and time convergence study of flow 
past savonius turbine blades using URANS based code is presented in [14]. The same boundary conditions are imposed, except for the inlet where no perturbation is set. The $\mathrm{k}-\omega$ SST turbulence model is chosen, which leads to fully turbulent boundary layer over the blade. The time step has been selected in order to achieve $C F L=u \Delta t / \Delta x$ less than or equal to 1 in the entire domain, and a second order backward Euler scheme has been choosen. First, the time average flow fields for the DNS and URANS computations are shown in Figure 18. It is noted that the flow in URANS calculation is already averaged by the turbulence model. The URANS computation does not predict the centrifugal instability and the development of mushroom like structures, which lead to quasi stationary flow in the fluid domain. The development of Görtler flow significantly increases the low speed region near the wall, up to the trailing edge of the blade. This can strongly modify the trailing edge vortex that plays an important role in the vortex shedding process of the turbine, and hence influences its overall performances.

The pressure and friction coefficients along the chord are shown in Figure 19. The absolute pressure level for URANS calculation is globally higher than its DNS counterpart. The two solutions are close to each other up to the breakdown of longitudinal streaks. When the flow gets unsteady and transitional for the DNS, the URANS calculation over-predict the pressure coefficient, which shows a maximum difference of about $C_{P}=0.2$ near the trailing edge. As far as the DNS solution is concerned, very few fluctuation is observed in the laminar region, whereas a maximum level of fluctuation is found in a region where the longitudinal streaks breakdown (i.e. between $\theta=80^{\circ}$ to $\theta=100^{\circ}$ ). The mechanism of breakdown obviously induces a 

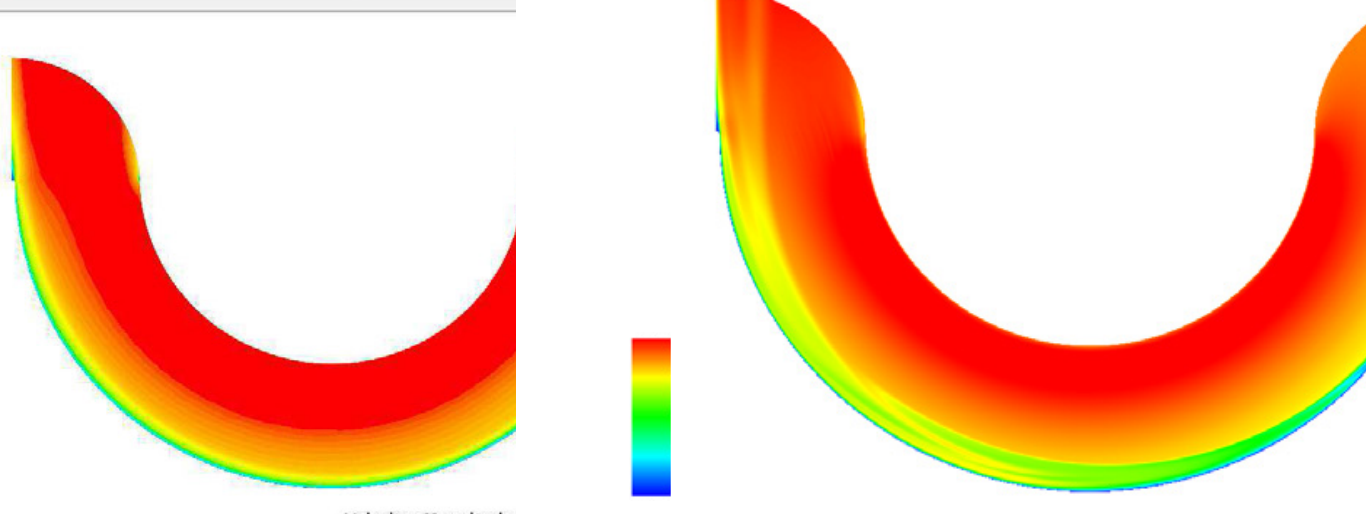

(b) DNS

Figure 18: Velocity field in the $(\xi, \eta)$ plane for $z=0.06$, comparison between URANS and DNS solutions.

strong and localized pressure jump. The fluctuations are then almost constant, whereas the average pressure coefficient presents a constant slope along $80 \%$ of the chord. As shown in Figure 1, a positive pressure coefficient on the returning blade produces a drag that act positively on the axis moment of the turbine, used in the energy conversion. Hence, its variation may directly influence the turbine power. The friction coefficient is very different for the two solutions. The reversed flow region near the leading edge induces negative friction coefficient for the DNS, whereas it is not predicted by the URANS based code. From the development of Görtler flow, the average friction coefficient predicted by DNS is almost two times higher than that of the URANS solution. This high level of shear is due to the development and the breakdown of longitudinal streaks in the DNS, compared to fully attached, turbulent boundary layer in the URANS calculation.

The time average lift and drag coefficients obtained for the URANS and 
DNS calculations are shown in Table 3. The lift and drag coefficients are defined as $C l=L /\left(0.5 \rho U_{\infty}^{2} c \times b\right)$ and $C d=D /\left(0.5 \rho U_{\infty}^{2} c \times b\right)$, where $c=2 R, b$, $L$ and $D$ are, respectively, the chord, the span, the lift and drag forces. As observed in this table as well as in Figure 19, the pressure drag presents a small variation because the larger pressure difference occurs near the trailing edge, where the surface normal is perpendicular to the drag. As a consequence, the pressure lift coefficient $C l_{p}$ is more affected, where $C l_{p}=0.02570$ is obtained for the URANS solution against $C l_{p}=0.0194$ for the DNS solution. The viscous drag is, however, strongly modified between the DNS and URANS solutions. After $\theta=90^{\circ}$, the high shear region associated with the development of longitudinal streaks, the breakdown and transition to turbulence increases the positive viscous drag. This significantly decreases the total drag which is oriented negatively. The viscous drag coefficient $C d_{v}=0.00041$ is obtained for the URANS solution against $C d_{v}=-0.0019$ for the DNS solution, which is three times more than that of the URANS solution and is in opposite direction. Therefore, it is clear that although URANS gives a good initial estimation SSWTs, DNS may be necessary to investigate the detailed flow physics and complex three-dimensional phenomena for such problems. Moreover, even if the differences in the total forces are weak in the present case, the authors believe that higher Reynolds numbers can induce significant differences in the performances due to larger transitional zone along the blade. 


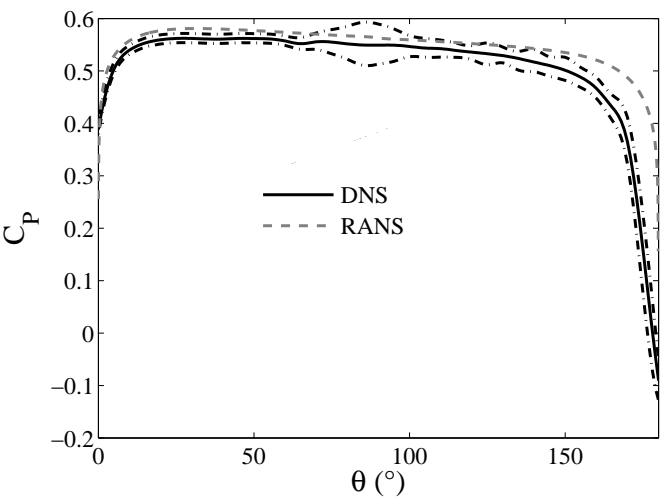

(a)

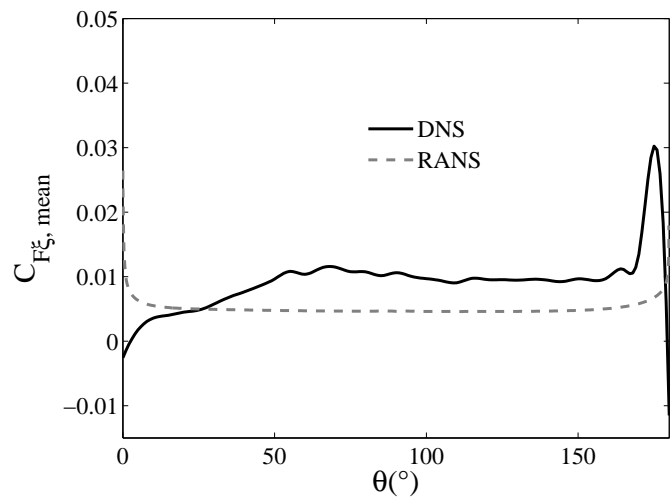

(b)

Figure 19: Comparison of the time averaged pressure and friction coefficient along the blade chord for the URANS the DNS solutions. (a) show the (-) mean values, (-.) fluctuations for the DNS solution.

\begin{tabular}{lccc}
\hline & pressure drag $C d_{p}$ & viscous drag $C d_{v}$ & total drag $C d_{\text {tot }}$ \\
\hline URANS & 0.55596 & 0.00041 & 0.55637 \\
\hline DNS & 0.5392 & -0.0019 & 0.5373 \\
\hline \hline & pressure lift $C l_{p}$ & viscous lift $C l_{v}$ & total lift $C l_{\text {tot }}$ \\
\hline URANS & 0.02570 & -0.00584 & 0.01990 \\
\hline DNS & 0.0194 & -0.0077 & 0.0117 \\
\hline
\end{tabular}

Table 3: Comparison lift and drag coefficient for the URANS and DNS solutions. 


\section{Conclusion}

In this paper, DNS calculations were performed to capture flow instabilities over the pressure side of a vertical axis Savonius-style wind turbine (SSWT). The flow domain is simplified in order to lead a DNS accuracy for a maximum Reynolds number of $R e_{\xi}=9 \times 10^{4}$. It is clear from this work that centrifugal instabilities occur along the blade, and trigger the boundary layer transition, which has not yet been considered in researches involving the improvement of SSWT performances.

It is observed that the boundary layer flow experiences classical centrifugal instabilities around the mid chord of the blade, where longitudinal streaks develop. The streaks then breakdown when secondary instabilities occur. The flow then governs by mushroom like structures followed by boundary layer transition to turbulence. Because of the relatively low Reynolds number induced by the small scale of the turbine, the mushroom structures occuring in the Görtler flow can compete with each other and maintain the laminar flow. The results show that this particular physic induces a significantly higher friction coefficient from the inception and, break down of the Görtler vortices until transition to turbulence.

The comparison with fully turbulent URANS calculation shows that the development of Görtler flow highly modify both the pressure and friction at the wall, which in turn modifies the viscous components of the drag and lift forces. It is noted that although several simplifications are made in the current DNS, the computational costs are still significant even when compared with the full domain URANS simulations. Nevertheless, unlike DNS, URANS cannot predict (i) the reverse flow at the leading edge, due to the 
fully turbulent flow hypothesis, (ii) the development of centrifugal instabilities as well as (iii) the thickness of low speed region near the wall. While the latter affects the trailing edge vortex and its consequence shedding that indirectly modify the turbine performance mainly by altering the reverse flow on the second blade, the two formers directly touch pressure and friction coefficients calculation. As examples, DNS shows (i) a negative friction coefficient at the leading edge, (ii) an average friction coefficient that is two times higher than URANS and (iii) a maximum difference of about $C_{p}=0.2$ in the pressure coefficient. It is also observed that the low speed region is highly increased in the DNS up to the trailing edge, which can strongly influence the well-known mechanism of trailing edge vortex shedding on the returning blade, which partially form the wake flow. Therefore, the accuracy reached by DNS along with the lack of data in the literature, necessitates the existence of the current work to provide more reliable data for such simple yet important geometry.

To the authors best knowledge, it is the very first attempt to perform DNS over a blade turbine, and to demonstrate that centrifugal instability can occurs on this kind of turbine. Even if very strong hypothesis has been taken, including no blade rotation, no suction side and constant velocity profile at the inlet; the results presented in this paper can help in the SSWT design and developments. The direct application to this study remains in the prediction of static performance, which determine the self starting capability of the turbine. More generally, as very few DNS studies were considered in the analysis of Görtler flow, this paper is also addressed to the centrifugal instability community. 
Possible extension of this study concerns the set up of realistic velocity profile at the inlet of the domain. This includes the implementation of isotropic turbulence models such as von-Kármán model, and the set up of mean velocity profiles that reproduces the gap flow between the blades, in particular for the leading edge flow detachment. To characterize these aspects, the investigation of the receptivity on the Görtler flow and transition mechanism would be necessary. Moreover, even if taken into account the full case of the turbine in rotation is not possible through DNS, the constant increase of computational power will allows to consider a more realistic case, such as simulating the flow over a full turbine blade, and increase the Reynolds number to investigate the performances for various operating conditions.

\section{Acknowledgements}

This work was performed using HPC resources of GENCI/IDRIS (Grant 2016-[100631]) at Orsay, France on the IBM Blue Gene/Q (Turing). Flow visualizations have been made using the open-source software VisIt [32].

[1] M. Bortolini, M. Gamberi, A. Graziani, R. Manzini, F. Pilati, Performance and viability analysis of small wind turbines in the european union, Renewable Energy 62 (2014) 629-639.

[2] P. Jaohindy, S. McTavish, F. Garde, A. Bastide, An analysis of the transient forces acting on savonius rotors with different aspect ratios, Renewable energy 55 (2013) 286-295. 
[3] S. Roy, U. K. Saha, Review on the numerical investigations into the design and development of savonius wind rotors, Renewable and Sustainable Energy Reviews 24 (2013) 73-83.

[4] J.-L. Menet, A double-step savonius rotor for local production of electricity: a design study, Renewable energy 29 (11) (2004) 1843-1862.

[5] K. Kacprzak, G. Liskiewicz, K. Sobczak, Numerical investigation of conventional and modified savonius wind turbines, Renewable energy 60 (2013) 578-585.

[6] D. Afungchui, B. Kamoun, A. Helali, Vortical structures in the wake of the savonius wind turbine by the discrete vortex method, Renewable Energy 69 (2014) 174-179.

[7] A. Ducoin, S. Roy, M. Shadloo, Direct numerical simulation of nonlinear secondary instabilities on the pressure side of a savonius-style wind turbine, in: ASME 2016 HT/FE/ICNMM, Heat Transfer, Fluids Engineering and Nanochannels, Microchannels, and Minichannels Conferences (ASME-FEDSM 2016), July 10-14, Washington, USA, 2016.

[8] M. Shadloo, A. Hadjadj, F. Hussain, Statistical behavior of supersonic turbulent boundary layers with heat transfer at $\mathrm{m}=2$, International Journal of Heat and Fluid Flow 53 (2015) 113-134.

[9] J. V. Akwa, H. A. Vielmo, A. P. Petry, A review on the performance of savonius wind turbines, Renewable and Sustainable Energy Reviews 16 (5) (2012) 3054-3064. 
[10] B. Plourde, J. Abraham, G. Mowry, W. Minkowycz, Simulations of three-dimensional vertical-axis turbines for communications applications, Wind Engineering 36 (4) (2012) 443-454.

[11] M. Mohamed, G. Janiga, E. Pap, D. Thévenin, Optimization of savonius turbines using an obstacle shielding the returning blade, Renewable Energy 35 (11) (2010) 2618-2626.

[12] S. Roy, U. K. Saha, Computational study to assess the influence of overlap ratio on static torque characteristics of a vertical axis wind turbine, Procedia Engineering 51 (2013) 694-702.

[13] J. V. Akwa, G. A. da Silva Junior, A. P. Petry, Discussion on the verification of the overlap ratio influence on performance coefficients of a savonius wind rotor using computational fluid dynamics, Renewable energy 38 (1) (2012) 141-149.

[14] S. Roy, A. Ducoin, Unsteady analysis on the instantaneous forces and moment arms acting on a novel savoniusstyle wind turbine, Energy Conversion and Management http://dx.doi.org/10.1016/j.enconman.2016.05.044 (2016) .

[15] J.-H. Lee, Y.-T. Lee, H.-C. Lim, Effect of twist angle on the performance of savonius wind turbine, Renewable Energy 89 (2016) 231-244.

[16] L.-U. Schrader, L. Brandt, T. A. Zaki, Receptivity, instability and breakdown of gortler flow, Journal of Fluids Mechanics 682 (2011) 362-396.

[17] J. Ren, S. Fu, Secondary instabilities of görtler vortices in high-speed boundary layer flows, Journal of Fluid Mechanics 781 (2015) 388-421. 
[18] T. Tandiono, S. Winoto, D. Shah, Spanwise velocity component in nonlinear region of görtler vortices, Physics of Fluids (1994-present) 25 (10) (2013) 104104.

[19] H. Peerhossaini, J. Wesfreid, On the inner structure of streamwise görtler rolls, International journal of heat and fluid flow 9 (1) (1988) 12-18.

[20] J. D. Swearingen, R. F. Blackwelder, The growth and breakdown of streamwise vortices in the presence of a wall, Journal of Fluid Mechanics 182 (1987) 255-290.

[21] J. Floryan, On the görtler instability of boundary layers, Progress in Aerospace Sciences 28 (3) (1991) 235-271.

[22] M. P. Schultz, R. J. Volino, Effects of concave curvature on boundary layer transition under high freestream turbulence conditions, Journal of Fluids Engineering 125 (1) (2003) 18-27.

[23] P. Fischer, J. Kruse, J. Mullen, H. Tufo, J. Lottes, S. Kerkemeier, Open source spectral element cfd solver, https://nek5000.mcs.anl.gov/index.php/MainPage. (2008).

[24] P. Fischer, J. Kruse, J. Mullen, H. Tufo, J. Lottes, S. Kerkemeier, Nek5000-open source spectral element cfd solver, Argonne National Laboratory, Mathematics and Computer Science Division, Argonne, IL, see https://nek5000. mcs. anl. gov/index. php/MainPage.

[25] A. Patera, A spectral element method for fluid dynamics: laminar flow in a channel expansion., J. Comput. Phys. 54 (1984) 468-488. 
[26] H. M. Tufo, P. F. Fischer, Terascale spectral element algorithms and implementations., In Proceedings of the ACM/IEEE SC99 Conference on High Performance Networking and Computing, Portland, U.S.A. (1999).

[27] H. M. Tufo, P. F. Fischer, Fast parallel direct solvers for coarse grid problems., Journal of Parallel Distributed Computing 61 (2) (2001) 151177.

[28] M. Deville, P. Fischer, E. Mund, High-Order methods for incompressible fluid flow, Cambridge University Press, 2002.

[29] G. Karniadakis, S. Sherwin, Spectral/hp element methods for computational fluid dynamics, Oxford science publications, 2005.

[30] J. Jiménez, P. Moin, The minimal flow unit in near-wall turbulence, Journal of Fluid Mechanics 225 (1991) 213-240.

[31] W. Tandiono, D. Shah, Wall shear stress in görtler vortex boundary layer flow, Physics of Fluids 21 (8) (2009) 084106-084106.

[32] H. Childs, E. Brugger, B. Whitlock, J. Meredith, S. Ahern, D. Pugmire, K. Biagas, M. Miller, C. Harrison, G. H. Weber, H. Krishnan, T. Fogal, A. Sanderson, C. Garth, E. W. Bethel, D. Camp, O. Rübel, M. Durant, J. M. Favre, P. Navrátil, VisIt: An End-User Tool For Visualizing and Analyzing Very Large Data, in: High Performance VisualizationEnabling Extreme-Scale Scientific Insight, 2012, pp. 357-372. 


\section{ACCEPTED MANUSCRIPT}

Direct Numerical Simulation is performed on a Savolius Style Wind Turbine blade

A high order spectral element method is used

Centrifugal instability occurs at the pressure side of the blade

The break down of Görtler vortices cause the flow to transit to turbulence

The pressure and friction distributions along the blade are altered by the transition phenomena 